\title{
A Semantic Web Service Oriented Framework for Adaptive Learning Environments
}

\author{
Stefan Dietze, Alessio Gugliotta, and John Domingue \\ Knowledge Media Institute \\ Open University \\ Milton Keynes, MK7 6AA, UK \\ \{s.dietze, a.gugliotta, j.b.domingue\}@open.ac.uk
}

\begin{abstract}
The current state of the art in supporting e-learning objectives is primarily based on providing a learner with learning content by using metadata standards. Due to this approach, several issues have to be taken into account - e. g. limited re-usability across different standards and learning contexts and high development costs. To overcome these issues, this paper describes an innovative semantic web service-oriented framework aimed at changing this data- and metadata-based paradigm to a highly dynamic service-oriented approach. Instead of providing a learner with static data, our approach is based on fulfilling learning objectives based on a dynamic supply of services. Therefore, we introduce a semantic layer architecture to abstract from existing learning data as well as process metadata standards by using Semantic Web Service (SWS) technology. Furthermore, our approach is based on abstract and reusable learning process models describing a learning process semantically as a composition of learning goals. Based on the formal semantic descriptions of learning goals as well as web services, services appropriate to achieve a specific learning goal can be selected, composed and invoked dynamically. This supports a high level of re-usability since a dynamic adaptation to different learning contexts and requirements of individual learners is achieved while utilizing standard-compliant learning applications. To illustrate the application of our approach, we describe a prototypical implementation utilizing the introduced approach based on the SWS framework WSMO.
\end{abstract}

Keywords: Semantic Web Services, Service oriented Architecture, WSMO, ELearning, IRS III, IMS Learning Design.

\section{Introduction}

Current approaches to support a learning objective are fundamentally based on providing a learner with appropriate learning content - the so called learning objects. Composite learning objects contain the learning resources - the physical data assets as well as a description of the learning process to be followed by the learner. The latter usually is based on existing metadata standards - IEEE LOM [9], ADL SCORM [1] - based on IMS Simple Sequencing - or IMS Learning Design (IMS LD) [15]. Due to the approach of allocating learning resources - whether services or data - at 
design-time of a learning process model, the actual learning context - known at runtime only - cannot be considered. This means, a new learning content package has to be developed for every different learning scenario or individual needs of specific learners. For instance, a package suiting the needs of a learner with specific preferences - e. g. his native language or technological platform - can suit only this specific requirements and cannot be reused across different learning contexts. The identified limitations (cf. [2], [16], [6]) can be summarized as follows:

\section{L1. Limited appropriateness and dynamic adaptability to actual learning contexts.} It is assumed that every learning objective occurs in a specific context which is defined by e. g. the preferences of the actual learner. Learning data is allocated at design-time what limits the appropriateness of the data to the actual learning context. Moreover, the use of data excludes the dynamic adaptability a priori. In parallel to data-centric approaches, analogous issues can also be observed with service-oriented approaches. However, in that case, these issues are related to the allocation of services only.

L2. Limited reusability across different learning contexts and metadata standards. Due to L1, for every different learning context having distinct requirements or learner needs a new learning content package has to be developed. Since metadata is described based on standard-specific specifications, an individual content package cannot be reused across different standards. Besides that, the current approach limits opportunities for reusing available learning data and service repositories.

L3. High development costs. Due to L1 and L2, high development costs have to be taken into account when developing standard-compliant E-Learning packages.

To overcome these issues, the approach described in this paper changes this data- and metadata-based paradigm to a dynamic service-oriented approach based on Semantic Web Service (SWS) technologies.

SWS are aimed at enabling a automatic discovery, composition and invocation of available Web services. Based on semantic descriptions of functional capabilities of available Web services, a SWS broker automatically selects and invokes Web services appropriate to achieve a given goal.

IRS-III [5], the Internet Reasoning Service, is an implementation of a SWS broker environment. It provides the representational and reasoning mechanisms, which enable the dynamic interoperability and orchestration between services as well as the mediation between their semantic concepts. IRS-III utilizes a SWS library based on the reference ontology Web Service Modelling Ontology (WSMO) [26] and the OCML representation language [7] to store semantic descriptions of Web services and knowledge domains.

WSMO is a formal ontology for describing the various aspects of services in order to enable the automation of Web service discovery, composition, mediation and invocation. The meta-model of WSMO defines four top level elements: Ontologies, Goals, Web Services and Mediators. Whereas Ontologies describe the terminology and its semantics used by Web Services, Web Service descriptions describe the capabilities and interfaces of a particular service. Moreover, Goals describe a task from a user perspective and Mediators handle data and process interoperability issues 
that arise when handling heterogeneous systems. As a result, we enable the automatic allocation of the adequate services at runtime - not only data - and the integration of a wide variety of learning resources - whether data or services.

The following section of the paper outlines the issues of current learning technologies which are addressed with this paper. Section 3 then describes our approach of using a SWS oriented architecture to support learning processes followed by a section describing our ontological framework. The fifth section explains a SWS oriented architecture implemented as a first prototype and the used development principles. Finally, we summarize the contributions of our work, draw a conclusion and provide an outlook to future work related to our approach.

\section{Related Work}

Several approaches follow the idea of using semantic Web or Web service technologies to provide dynamic as well as personalized support for learning objectives.

To quote a few examples, [16] as well as [3] are concerned with bridging learning contexts and resources by introducing semantic learning context descriptions. This allows the adaptation to different contexts based on reasoning over the provided context ontologies, but does not provide solutions for building complex adaptive learning applications by reusing distributed learning functionalities. Moreover, [16] is entirely based on IMS LD.

[4] follows the idea of using a dedicated personalization web service which makes use of semantic learning object descriptions to identify and provide appropriate learning content. Integration of several distributed learning services or service allocation at runtime is not within the scope of this approach. The related research on a Personal Reader Framework (PRF) introduced in [8], [13] and [14] allows a mediaton between different services based on a socalled "connector service". The composition of complex learning applications based on distributed services is not within the scope of the PRF.

The work described in [22], [23] utilize semantic web as well as web service technologies to enable adaptation to different learning contexts by introducing a matching mechanism to map between a context and available learning data. However, neither it considers approaches for automatic service discovery nor it is based on common standards. Hence, the reuse and automatic allocation of a variety of services or the mediation between different metadata standards is not supported. These issues apply to the idea of "Smart Spaces" for learning as well (cf. [24]).

Whereas the majority of the described approaches enable context-adaptation based on runtime allocation of learning data, all of them do not enable the automatic allocation of learning functionalities neither it does enable the integration of new functionalities based on open standards. Nevertheless, all approaches do not envisage mappings between different learning metadata standards to enable interoperability not only between learning contexts but also between platforms and metadata standards. 


\section{Semantic Web Service Based E-Learning Applications: Vision and Approach}

This section describes our vision as well as the approach to support e-learning based on semantic web services.

\subsection{Vision: Context-Adaptation Through Automatic Service Selection and Invocation}

To overcome the limitations described above, we consider the automatic allocation and invocation of functionalities at runtime. A typical learning related service functionality provides the learner for instance with appropriate learning content or topic-specific discussion facilities. Learning processes are described semantically in terms of a composition of user objectives (goals) and abstract from specific data and metadata standards. When a specific learning goal has to be achieved, the most adequate functionality is selected and invoked dynamically regarding the demands and requirements of the actual specific context. This enables a highly dynamic adaptation to different learning contexts and learner needs.

This vision is radically distinctive to the current state of the art in this area, since it shifts from a data- and metadata-centric paradigm to a context-adaptive serviceoriented approach. Moreover, using adequate mappings, our standard-independent process models can be translated into existing metadata standards in order to enable a reuse within existing standard-compliant runtime environments.

Addressing the limitations L1 and L2 identified in Section 0, we consequently reduce the efforts of creating learning process models (L3): one unique learning process model can adapt dynamically to different process contexts and can be translated into different process metadata standards.

\subsection{Approach: Semantic Abstraction from Process Metadata, Functionalities and Data}

Our approach is fundamentally based on utilizing SWS technologies to realize the following principles. To support these principles, we introduce several layers as well as a mapping between them in order to achieve a gradual abstraction (Figure 1).

1. Abstraction from Learning Data and Functionalities. To abstract from existing learning data and content we consider a Web Service Layer. It operates on top of the data and exposes the functionalities appropriate to fulfill specific learning objectives. This first step enables a dynamic supply of appropriate learning data to suit a specific context and objective. Web services at this layer may make use of semantic descriptions of available learning data. In order to abstract from these functionalities (Web services), we introduce an additional layer - the Semantic Web Service Layer. This layer enables the automatic selection, composition and invocation of appropriate Web services for a specific learning context. This is achieved on the basis of formal semantic, declarative descriptions of the capabilities of available services which enable the dynamic matching of service capabilities to specific user goals. 
2. Abstraction from Learning Process Metadata. A first layer concerned with the abstraction from current learning process metadata standards is the Semantic Learning Process Model Layer. It allows the description of processes within the domain of E-Learning in terms of higher level domain concepts - e. g. learning goals, learners or learning contexts. This layer is mapped to semantic representations of current learning metadata standards in order to enable the interoperability between different standards. To achieve a further abstraction from domain specific process models - whether it is e. g. a learning process, a business process or a communication process - we consider an upper level process model layer - Semantic Process Model Layer. For instance, this layer supports the mapping between learning objectives and business objectives to support all kind of organizational processes.

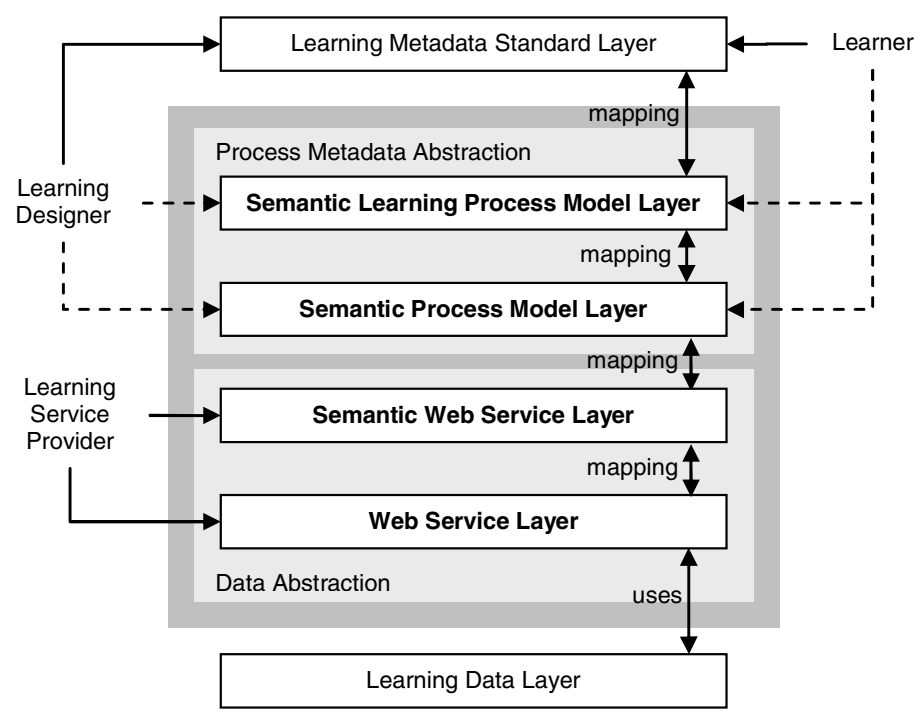

Fig. 1. Semantic layer architecture for supporting learning processes through SWS

Based on mappings between the described layers, upper level layers can utilize information at lower level layers. In particular, we consider mappings between a learning objective and a WSMO goal to enable the automatic discovery and invocation of a Web service (Web Service Layer) from, for instance, a standardcompliant learning application (Learning Application Standard Layer). As a result, a dynamic adaptation to individual demands of a learner within a specific learning context is achieved by using existing standard-compliant learning applications. It is important to note, that we explicitly consider mappings not only between multiple semantic layers but also within a specific semantic layer. 


\section{The Ontological Framework}

This section describes an ontological framework aimed at implementing the introduced semantic layers.

\subsection{Staged Ontological Mapping}

To implement the described semantic layer architecture, we follow an approach of a staged ontological mapping between semantic models of a process at different levels of abstraction. Therefore, our approach considers different ontologies aimed at providing abstract semantic descriptions of data as well as processes.

The following figure gives an overview of the main ontological representations considered in our approach as well as their relationships:

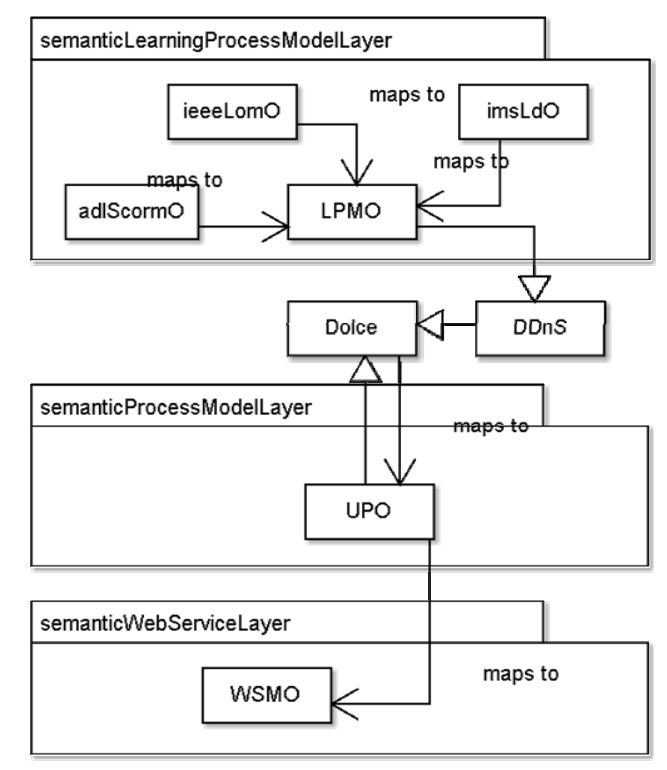

Fig. 2. Conceptual overview of proposed ontological framework

To enable mappings between different learning metadata standards, a higher level ontology is introduced, to model the learning process from a general point of view independent from any supported platform or learning technology standard. This Learning Process Modelling Ontology (LPMO) implements the Semantic Learning Process Model Layer and is mapped to ontological representations of learning process models based on current e-learning metadata standards. Currently, representations of the following metadata standards are foreseen: adlScormO (The ADL SCORM 2004 Ontology); imsLdO (The IMS Learning Design Ontology); ieeeLomO (The IEEE LOM Ontology). 
The next level in our staged mapping approach abstracts from the specialised process - e.g. learning process or business process - to a general process ontology. This is the Upper Process Ontology (UPO) which implements our Semantic Process Model Layer and is currently being developed as part of the SUPER project [25]. The UPO enables the description of a process independent from its specific purpose and can be mapped to domain specific process ontologies, e. g. the LPMO. In order to enable a high level of interoperability of our ontologies, we intend to align the LPMO as well as the UPO to the DOLCE foundational ontology [12] as well as to the DOLCE Descriptions and Situations ontology (DDnS) [11].

Furthermore, the UPO is mapped to the WSMO standard. Therefore, a gradual mapping between a standard learning application and WSMO entities is achieved based on these ontologies. It has to be highlighted, that our ontological architecture explicitly considers mappings not only between several semantic layers but also within a specific semantic layer. This enables for example the mapping of our LPMO concepts to other existing semantic descriptions of learning related concepts. Furthermore, it has to be taken into account that the proposed ontologies are currently implemented only partially, since this work is ongoing research at the moment.

\subsection{Semantic Learning Process Model Layer}

From an e-learning perspective, the LPMO has to be perceived as the central ontology within our architecture, since it describes the semantics of a learning process from a

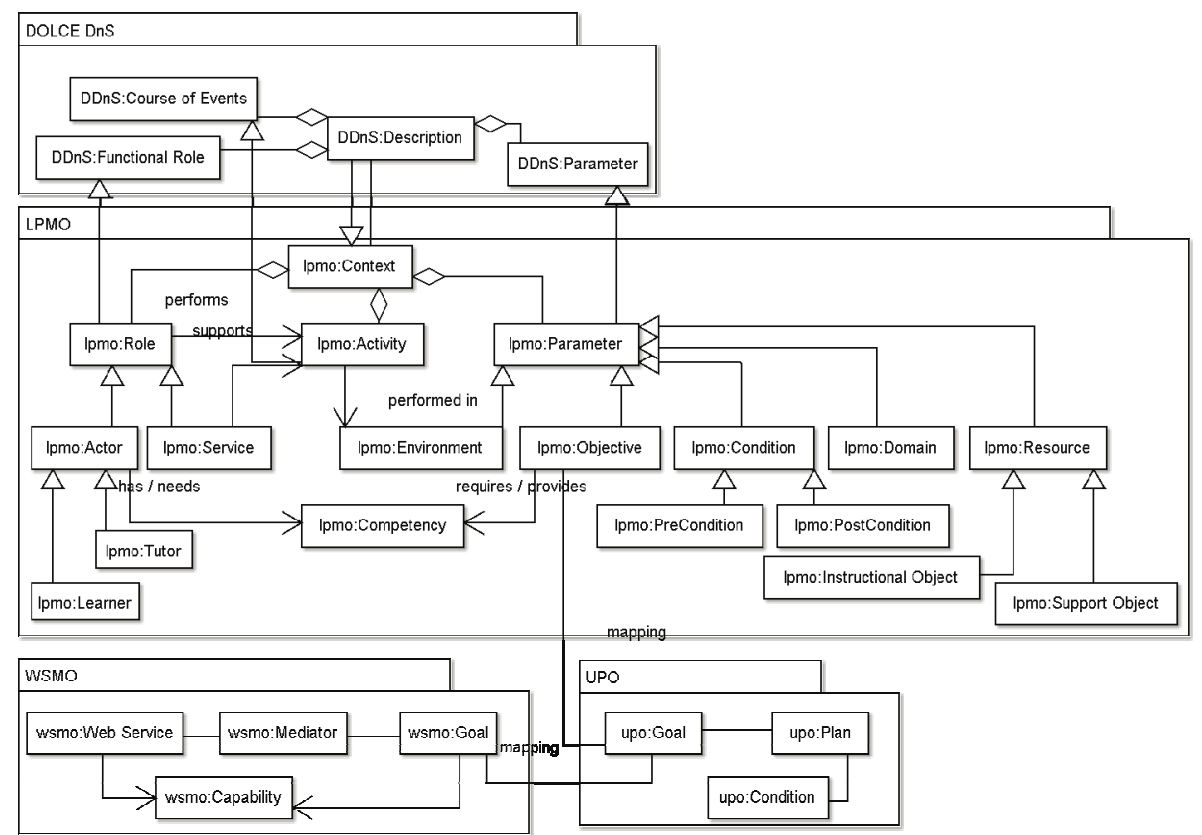

Fig. 3. Conceptual model of parts of the LPMO and key mappings to the UPO and the WSMO framework 
general point of view and independent from any supported platform or learning technology standard. Figure 3 depicts an extract of the proposed LPMO containing some of its main concepts as well as some mappings to some key concepts within different semantic layers.

As shown below, a learning objective as defined in the LPMO is mapped to a upo:Goal - which represents a central concept within the Semantic Process Model Layer. This concept is furthermore mapped to the wsmo:Goal concept which represents one of the main concepts of the Semantic Web Service Layer and enables the mapping and matching of appropriate web services. Besides the proposed mappings between several semantic layers, mappings are also considered within a specific layer to enable a wide applicability of our approach. E. g. semantic concepts of our LPMO can be mapped to other existing semantic concepts representing learning-related entities within different approaches - e. g. learning process modules as defined in [19], [17].

\section{A SWS Based Framework for E-Learning - Prototype Application Based on IMS Learning Design and WSMO}

In order to validate the technical feasibility of the described approach, a first prototype was implemented. In this section, we describe an application based on IMS Learning Design as well as the WSMO framework. The application implements an initial use case by utilizing the semantic layers and fundamental concepts as introduced in 3.2.

\subsection{Use Case: An Adaptive IMS LD Learning Package to Support Language Learning}

Within our supported scenario, several learners request to learn different languages: English, German and Italian. It is assumed, that all learners have different preferences - e.g. their spoken native language or technical environment. Following the current approach of creating standard-compliant learning content packages, for every individual learner a specific package would have to be created in order to achieve a high level of appropriateness to the individual learner needs. Based on our application, we enable all learners to use the same learning content package - an IMS LD compliant content package. This is achieved by enabling a dynamic adaptation to the individual learner requirements based on a dynamic selection and invocation of semantic web services at runtime.

For example, a learner is authenticated as a person with the native language "English" and wants to learn the language "German". By following a learning process as defined in the content package, the learner will be provided with learning content appropriate to his specific native language as well as his current learning objective an English-based online learning unit aimed at teaching the German language. Due to the dynamic adaptation at runtime, the standard-compliant learning process could suit 
all kind of different individual requirements. Since our approach is fundamentally based on the principles described in section 0 , this scenario could be extended in the future to achieve a dynamic adaptation to all kind of different learning contexts or learner requirements.

\subsection{A SWS Oriented Architecture}

To implement a software architecture aimed at supporting our semantic web service based approach, several dedicated software layers have to be provided. The following figure illustrates the SWS oriented architecture for e-learning which is utilized in the prototype application:

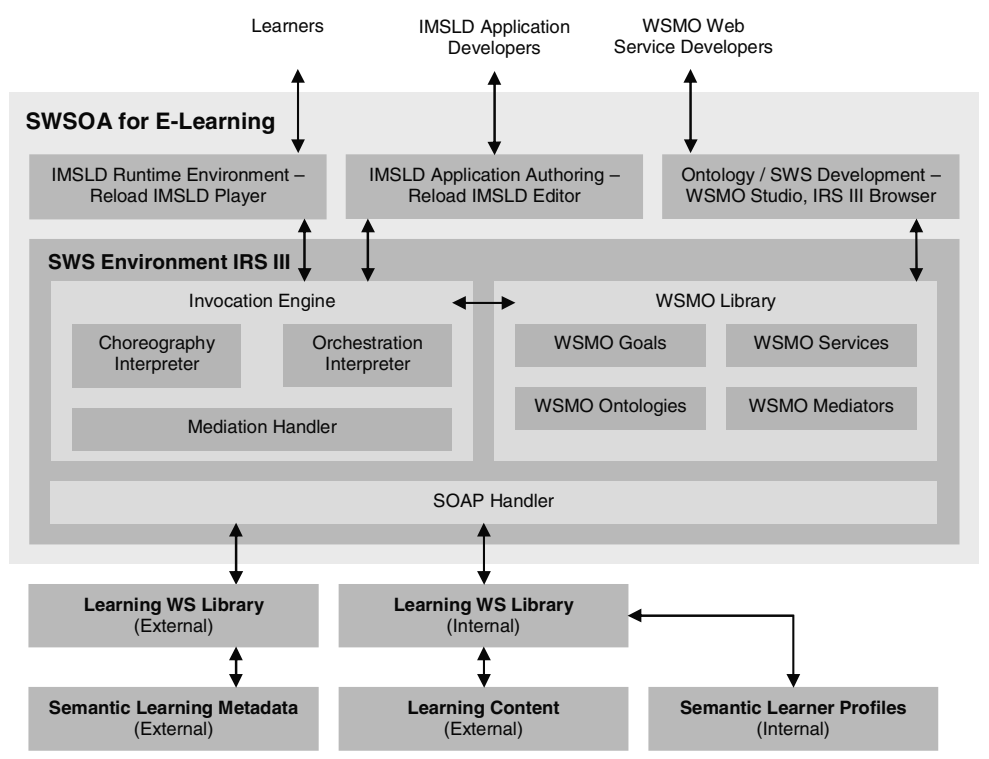

Fig. 4. SWS-based software architecture as utilized in the prototype application

The architecture depicted above is fundamentally based on the semantic layers described in section 3.2. In the figure, a SWS broker based on the WSMO framework serves as foundation for a dynamic selection, composition and invocation of web services. Services are distributed across different external repositories and provide functionalities based on existing learning data and metadata repositories. In addition, several user interfaces for developing and presenting learning applications as well as for developing formal semantic descriptions of web services are utilized. Our current implementation makes use of standard runtime environments and implements a SWS oriented architecture based on these infrastructural components. For WSMO runtime processing as well as development environment for WSMO, the SWS broker IRS III 
[5] is used, whereas editing and runtime processing of IMS LD is supported by the Reload Learning Design Editor and Player [21].

\subsection{Implementation Approach}

To support the described scenario based on an SWS-based approach the following items had to be provided:

- An IMS LD-compliant content package describing the learning activities and objectives

- Web services able to achieve the objectives

- Semantic descriptions of available services based on WSMO

- Ontologies implementing the semantic layers as described above

- Mappings between the semantic layers as well as the IMS LD standard.

As starting point, initial semantic representations of the LPMO, IMS LD as well as utilized content objects were provided in terms of OCML [7] ontologies to implement the Semantic Learning Process Model Layer. To support individual learner preferences, we particularly consider semantic learner profiles which describe the native language of every learner.

The web services utilized in this demonstrator were partly developed within the LUISA project [18] which is aimed at providing innovative learning content management technologies based on a SWS oriented architecture. Additional services were provided to support e. g. the authentication of the learner, the retrieval of semantic learner profiles or the retrieval of learning content. In addition, the mappings between the semantic layers were implemented as web services - e. g. a mapping between the Semantic Web Service Layer and the Semantic Learning Process Model Layer.

Besides that, a learning process was described based on the IMS LD standard and included into a IMS Content Package. The learning process in our example defines some learning activities (imsld:Activities) as well as corresponding sequencing information. Instead of grounding these activities to static learning data, no static resources were associated with this learning process. In contrast, only references to the described WSMO-Goals were associated with every learning activity within the IMS LD metadata. At runtime, a WSMO-Goal then dynamically invokes a WSMOweb service which shows the appropriate capabilities to achieve the specific goal. The mapping between the IMS LD metadata and appropriate WSMO-Goals was achieved by associating IMS LD learning activities with HTTP-references to a web applet enabling to request the achievement of a specific WSMO-goal from the SWS broker.

\subsection{Ontological Mappings}

As described above, we created mappings between the initial implementations of semantic representations of the IMS LD standard, the LPMO and WSMO. This 
includes e. g. a mapping between the lpmo:Objective and the objective description used within the IMS LD metadata (imsld:Objective). Furthermore, semantic learning object descriptions based on the LPMO are mapped to learning content provided by the Open Learn Project [20] based on an initial ontology representing this specific learning content objects. Besides that, a web service implements the mapping between the language of a content object (ol:Language) and the native language of the learner (lpmo:Language).

It has to be highlighted, that our current prototype does not implement the mapping to the UPO. Instead of that, our learning process was mapped directly to WSMO, since the UPO currently is not supported by any software RTE. The following figure depicts the main ontological mappings as implemented in our prototype:

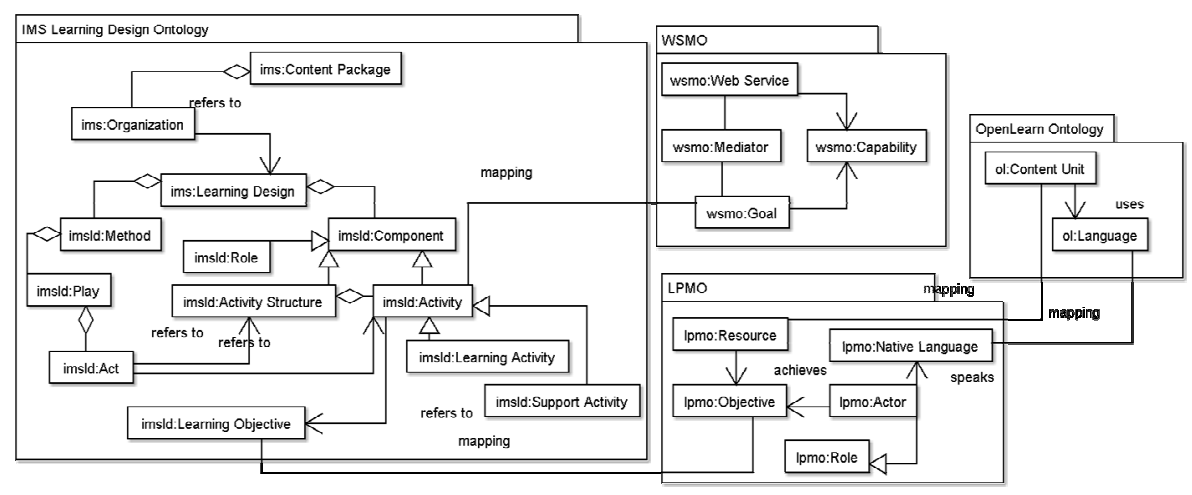

Fig. 5. Ontological mappings implemented and utilized in the prototype

\subsection{Dynamic Adaptation at Runtime}

In our example scenario, several web services are invoked to retrieve semantic learning metadata, learner profile descriptions and e-learning content as well as to map between different semantic concepts. An initial service first authenticates the learner and retrieves the semantic learner profile description. After providing an individual objective, our application dynamically selects and invokes semantic web services appropriate to the individual learner preferences and his specific objectives as defined in the IMS LD metadata.

For example, a learner is authenticated as an English-speaking person (lpmo:Language=English) and uses an IMSLD package to learn the language German. Therefore, an imsld:Activity with the imsld:Objective "Learn German" is mapped to a WSMO-goal to achieve this learning activity. This triggers the selection, orchestration and invocation of different web services to achieve the described mappings and to retrieve appropriate learning content. The following OCML code 
listing shows the partial capability description of a web service able to provide learning content to teach the language German:

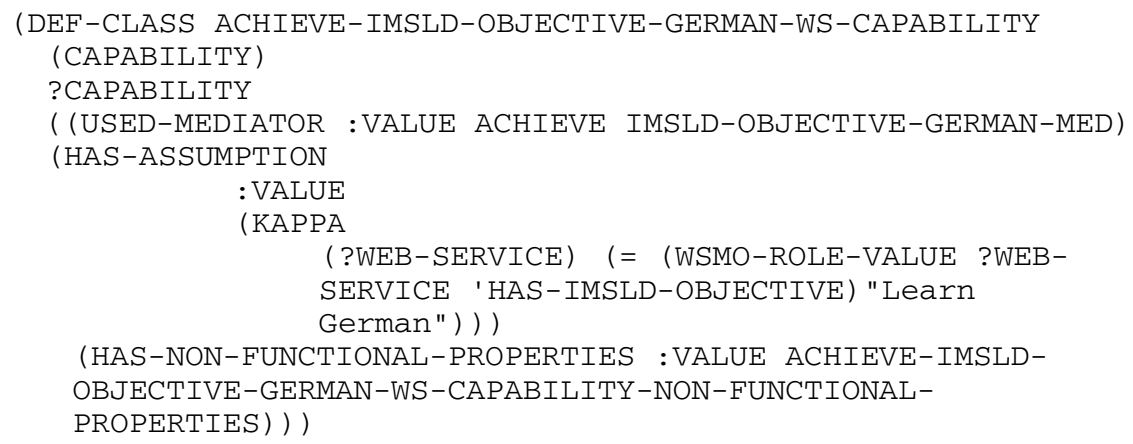

Listing 1. Partial source code of a web service capability description

In the listing above, a WSMO description defines the assumption of a web service that the objective provided by the IMS LD package has the Value "Learn German". The imsld-Objective is furthermore mapped to the lpmo:Objective concept in order to invoke another service for retrieving semantic metadata of an appropriate learning object based on the lpmo:Objective. The retrieved object identifier is used to obtain an Open Learn object appropriate to the individual language of the learner and its current objective. An appropriate learning object is then presented dynamically in the IMS LD runtime environment.

Figure 6 depicts a screenshot of the Reload IMS LD Player while presenting the developed standard-compliant IMS Content Package and dynamically invoking SWS appropriate to fulfill the given learning objective "Learn German".

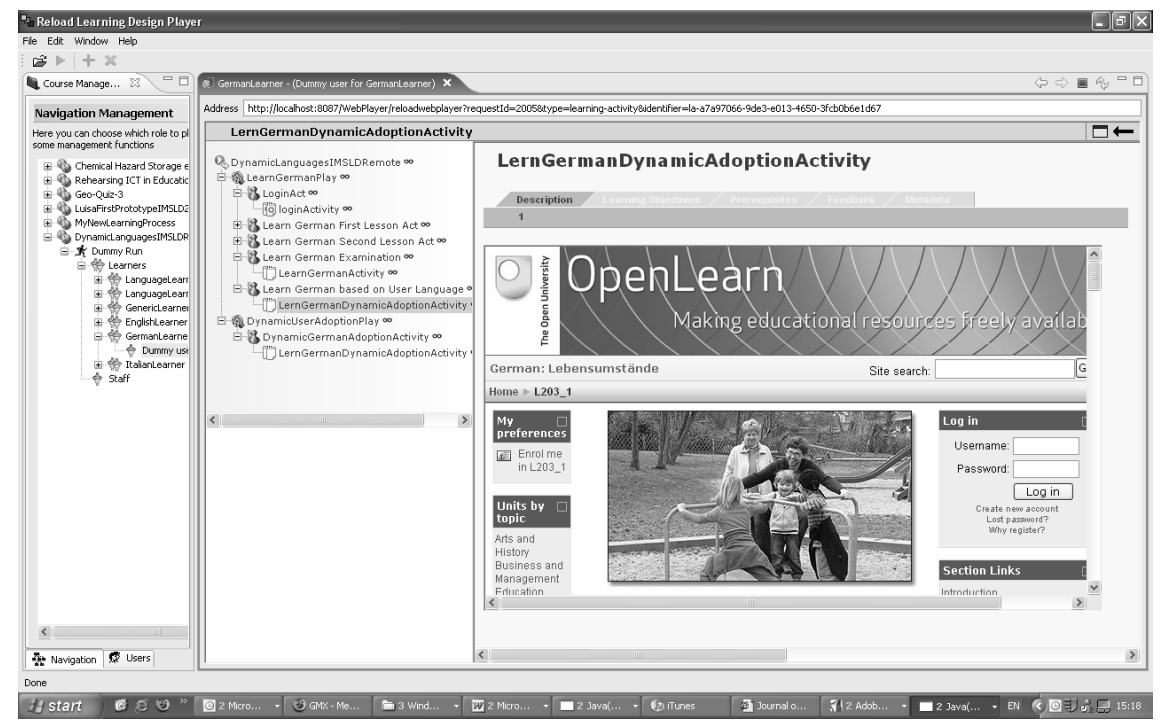

Fig. 6. Reload IMS Learning Design Player while dynamically invoking SWS for e-learning 
The current prototype implements the basic approach of a standard-compliant SWSOA for e-learning as described here, and will be extended in the future in order to address existing limitations. Furthermore, the described approach was already applied to another e-learning standard - ADL SCORM 2004.

\section{Conclusions}

Our approach - the support of learning objectives based on a dynamic invocation of SWS at runtime of a learning process model - follows an innovative approach and is distinctive to the current state of the art in this area. By using semantic web as well as SWS technology this approach overcomes the limitations described in Section 0 and provides a high level of openness to reuse existing service and data repositories. Based on existing standards - SWS as well as E-Learning standards - new learning data as well as application functionalities can be integrated by our SWS oriented architecture. Furthermore, a high level of standard-compliancy and re-usability within existing runtime environments is supported. In particular, the following contributions should be taken into account:

- Dynamic adaptation to specific learning contexts at runtime

- Reuse and integration of available learning resources - services and data

- Automatic allocation of learning resources based on comprehensive semantics

- High reusability across learning contexts

- Platform- and standard-independence

- Decrease of development costs

Furthermore, our approach can lead to contributions for developing domainspecific SWS applications in general, since we consider mappings between the WSMO standard and higher-level process modeling as well as learning process modeling standards. This enables the development of complex SWS based applications and therefore several benefits are envisaged:

- Re-usability of SWS based applications based on semantic mappings with existing process metadata standards

- Utilization of established standard-compliant software environments to implement complex SWS based architectures

Since our framework is developed only in parts currently, next steps have to be concerned with the implementation of complete ontological representations of the introduced semantic layers as well as of current E-Learning metadata standards and their mappings. For example, currently the Semantic Process Model Layer is not used and semantic mappings between the Learning Process Model Ontology and available process metadata standards are only developed in extracts. Nevertheless, the availability of appropriate Web services aimed at supporting specific process objectives has to be perceived as an important prerequisite for developing SWS based applications. To provide more valid quantifications of the expected benefits, further case studies are needed to illustrate the formalized measurements introduced in the 
sections above. Besides that, future work could also be concerned with the mapping of semantic process models across different process dimensions - e. g. business processes or learning processes to enable a complete integration of a SWSOA in an organizational process environment.

\section{References}

1. Advanced Distributed Learning (ADL) SCORM 2004 Specification (http://www.adlnet.org).

2. Amorim, R. R., Lama, M., Sánchez, E., Riera, A., \& Vila, X. A. (2006). A Learning Design Ontology based on the IMS Specification. Journal of Educational Technology \& Society, 9 (1), 38-57.

3. M. Baldoni, C. Baroglio, V. Patti, and L. Torasso. Using a rational agent in an adaptive web-based tutoring system. In Proc. of the Workshop on Adaptive Systems for Web-Based Education, 2nd Int. Conf. on Adaptive Hypermedia and Adaptive Web-based Systems, pages 43-55, Malaga, Spain, 2002.

4. M. Baldoni, C. Baroglio, I. Brunkhorst, N. Henze, E. Marengo and V. Patti: A Personalization Service for Curriculum Planning. ABIS 2006 - 14th Workshop on Adaptivity and User Modeling in Interactive Systems, Hildesheim, October 9-11 2006.

5. Cabral, L., Domingue, J., Galizia, S., Gugliotta, A., Norton, B., Tanasescu, V., Pedrinaci, C.: IRS-III: A Broker for Semantic Web Services based Applications. In proceedings of the $5^{\text {th }}$ International Semantic Web Conference (ISWC 2006), Athens, USA (2006).

6. Collis, B. and Strijker, A. (2004). Technology and Human Issues in Reusing Learning Objects. Journal of Interactive Media in Education, 2004 (4). Special Issue on the Educational Semantic Web [www- jime.open.ac.uk/2004/4].

7. J. Domingue, E. Motta and O. Corcho Garcia (1999). Knowledge Modelling in WebOnto and OCML: A User Guide, available from: http://kmi.open.ac.uk/projects/webonto/user_guide.2.4.pdf.

8. Dolog P., Henze, N., Nejdl, W., Sintek, M., Personalization in Distributed elearning Environments, In Proc. Of WWW2004 - The $13^{\text {th }}$ international World Wide Web Conference, 2004.

9. Duval, E. (2002). 1484.12.1 IEEE Standard for learning Object Metadata, IEEE Learning Technology Standards Committee, http://ltsc.ieee.org/wg12/.

10. Fischer, G. and Ostwald, J. (2001). Knowledge Management: Problems, Promises, Realities, and Challenges, IEEE Intelligent Systems, 16-1(60-72). http://citeseer.ist.psu.edu/489331.html.

11. Gangemi, A., and Mika, P. Understanding the Semantic Web through Descriptions and Situations. In Meersman, R.; Tari, Z.; and et al., D. S., eds., Proceedings of the On The Move Federated Conferences (OTM'03), LNCS. Springer Verlag.

12. Gangemi, A., Guarino, N., Masolo, C., Oltramari, A., Schneider, L.: Sweetening Ontologies with DOLCE. In: A. Gómez-Pérez, V. Richard Benjamins (Eds.) Knowledge Engineering and Knowledge Management. Ontologies and the Semantic Web: 13th International Conference, EKAW 2002, Siguenza, Spain, October 1-4, 2002.

13. Henze, N., Personalized e-Learning in the Semantic Web. Extended version of 4. International Journal of Emerging Technologies in Learning (iJET), Vol. 1, No. 1 (2006).

14. Nicola Henze, Peter Dolog, and Wolfgang Nejdl: Reasoning and Ontologies for Personalized E-Learning. Educational Technology \& Society, 2004, Vol. 7, Issue 4.

15. IMS Learning Design Specification (http://www.imsglobal.org). 
16. Knight, C., Gašević, D., \& Richards, G. (2006). An Ontology-Based Framework for Bridging Learning Design and Learning Content. Journal of Educational Technology \& Society, 9 (1), 23-37.

17. Koper, R. (2004). Use of the Semantic Web to Solve Some Basic Problems in Education: Increase Flexible, Distributed Lifelong Learning, Decrease Teacher's Workload. Journal of Interactive Media in Education, 2004 (6). Special Issue on the Educational Semantic Web. ISSN:1365-893X [www-jime.open.ac.uk/2004/6].

18. LUISA Project - Learning Content Management System Using Innovative Semantic Web Services Architecture (http://www.luisa-project.eu/www/).

19. Naeve, A., Sicilia, M. A. (2006), Learning Processes and processing learning: from organizational needs to learning designs, ADALE workshop at the Adaptive Hypermedia conference, Dublin, June 20-23, 2006.

20. Open Learn Project: Online Educational Resources (http://openlearn.open.ac.uk/).

21. Reload Project (http://www.reload.ac.uk/).

22. Schmidt, A., Winterhalter, C. User Context Aware Delivery of E-Learning Material: Approach and Architecture, Journal of Universal Computer Science (JUCS) vol.10, no.1, January 2004.

23. Schmidt, A., Bridging the Gap Between E-Learning and Knowledge Management with Context-Aware Corporate Learning (Extended Version), In: Professional Knowledge Management (WM 2005), Post Proceedings, Springer, 2005.

24. Simon, B., Dolog., P., Miklós, Z., Olmedilla, D. and Sintek, M. (2004). Conceptualising Smart Spaces for Learning. Journal of Interactive Media in Education, 2004 (9). Special Issue on the Educational Semantic Web. ISSN:1365-893X [http://wwwjime.open.ac.uk/2004/9].

25. SUPER - Semantics Utilized for Process Management within and between Enterprises (http://www.ip-super.org/).

26. WSMO Working Group, D2v1.0: Web Service Modeling Ontology (WSMO). WSMO Working Draft, (2004). (http://www.wsmo.org/2004/d2/v1.0/). 